\title{
Optimization of CABRI power transients with the SPARTE code and the URANIE uncertainty platform
}

\author{
O. Clamens ${ }^{\mathrm{a}, *}$, J-P. Hudelot ${ }^{\mathrm{a}}$, P. Blaise ${ }^{\mathrm{a}}$, J. Lecerf ${ }^{\mathrm{a}}$, B. Duc ${ }^{\mathrm{a}}$, Y. Garnier ${ }^{\mathrm{a}}$, \\ B. Biard $^{\mathrm{b}}$ \\ ${ }^{a}$ CEA, DEN, DER/SPESI/LP2E, Cadarache, F-13108 Saint Paul les Durance, France \\ ${ }^{b} I R S N / P S N-R E S / S E R E X / L 2 E P$, Cadarache, BP3 13115 Saint-Paul-lez-Durance Cedex, \\ France
}

\begin{abstract}
In a Pressurized Water Reactor (PWR), the rod ejection is a design basis accident for uncontrolled evolution of the nuclear reaction. In case of failure of a rod mechanism, the rod ejection is caused by the pressure differential between the primary loop (155 bar) and the confinement's enclosure (atmospheric pressure). It leads to a local power transient and a fast fuel temperature increase. The power transient is limited by the reactivity feedbacks before the automatic reactor shutdown.

The CABRI experimental pulsed reactor is funded by the French Nuclear Safety and Radioprotection Institute (IRSN) and is operated by CEA at the Cadarache research center. It is designed to study fuel rods behavior under Reactivity Initiated Accident (RIA) conditions. The tested fuel rod is placed at the center of the CABRI core, inside a pressurized water loop reproducing PWR conditions. CABRI is a pool type reactor, made of $1487 \mathrm{UO}_{2}$ fuel rods and controlled by 6 Hafnium control rods. A specific device allows the fast depressurization of ${ }^{3} \mathrm{He}$ contained in 4 transient rods to reproduce control rods ejection conditions.

Based on a BEPU approach, we developed a tool, named SPARTE, for CABRI power transients calculation. This tool is based on point kinetics, simplified thermal-hydraulics and thermal-mechanics. It computes the global behavior of the core by the calculation of a mean fuel rod. It includes models of reactivity insertion specific to the CABRI transient rods system, variable
\end{abstract}

\footnotetext{
${ }^{*}$ Corresponding author

Email address: olivier.clamens@cea.fr (O. Clamens)
} 
kinetics parameters and variable Doppler coefficient. This code is validated on the basis of 66 CABRI start-up power transients realized during the first quarter of 2017.

One goal of the SPARTE code is to be used for the prediction of future CABRI power transients. This paper focuses on methods for optimizing a specific CABRI power transient $(F W H M \simeq 30 \mathrm{~ms}$, Deposited energy $\simeq$ $130 \mathrm{MJ}$ ) using the target characteristics of the pulse. The selection of a method may help the experimentalists and the operation team to minimize the number of "white" power transients to perform before the final test with the fuel sample. The optimization can lead to different results, that can be ranked according to their projected uncertainties.

Different optimization methods are tested and compared in this paper. The Subplex method based on reiterations of the Nelder-Mead algorithm (simplex method) was selected for its high precision. Indeed, the CABRI power transients are not completely reproducible and present some uncertainties linked to the test parameters. This article focuses on the uncertainties propagation in order to identify and select the parameters that minimize the output uncertainties. The results are very satisfactory and lead to several optimized scenarios that will be tested during the next qualification test campaign.

Keywords: RIA, CABRI, Multi-physics, Optimization, Uncertainties

\section{Introduction}

The CABRI International Program (CIP) operated and managed by IRSN is dedicated to the study of irradiated PWR fuel rods behavior under RIA conditions. The target power transients are defined by their Full Width at Half Maximum (FWHM) and the energy deposited inside the tested fuel rod. The experimentalist team role is to reach the target conditions defined by the CIP project while respecting the safety domain of the CABRI core. The power transients characteristics are controlled by adjusting the parameters of the reactivity injection.

The reactivity is injected by a unique transient rods system. The CABRI transient rods system is made of the following main components (see Figure 2):

- 4 fuel assemblies (7x7 pins) equipped on their periphery with 24 tubes instead of 24 fuel rods. These tubes are connected together in the upper 


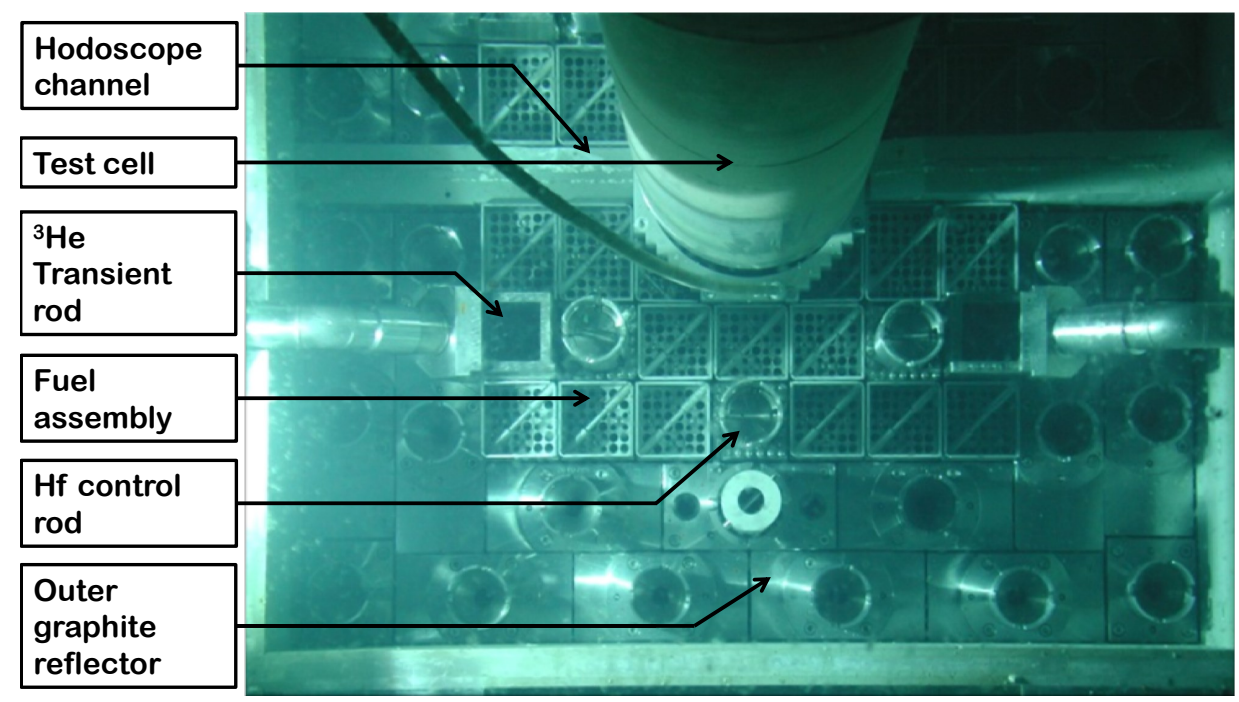

Figure 1: Radial cut of the CABRI reactor core

part of each assembly in order to join a collecting line leading to a main collector.

- From the top of this collector, two flow channels (low and high flow rates) lead to a $1000 l$ discharge tank set under vacuum before operation. Both channels are equipped with a fast-opening valve (respectively with small and large diameters) followed by a controlled valve.

- A specific control device that triggers the different orders of the experimental sequence as for the opening time of the two fast-opening valves and the shutdown of the reactor Hafnium control rods.

The transient rods depressurization causes the absorber ejection that induces a reactivity injection reaching up to $3.9 \$$ in few milliseconds. The characteristics of the transient (maximum power, Full Width at Half Maximum (FWHM) and energy deposit) depend on the experimental sequence applied to the fast valves and on the adjustment of the associated controlled valves. The control rods drop instant is adjusted in order to master the total core energy deposit. Short FWHM power transients $(\simeq 10 \mathrm{~ms})$, so called "natural transients", will be generated by the fast opening of the unique high flow rate channel.

In order to be representative of other LWR accidental conditions, an 


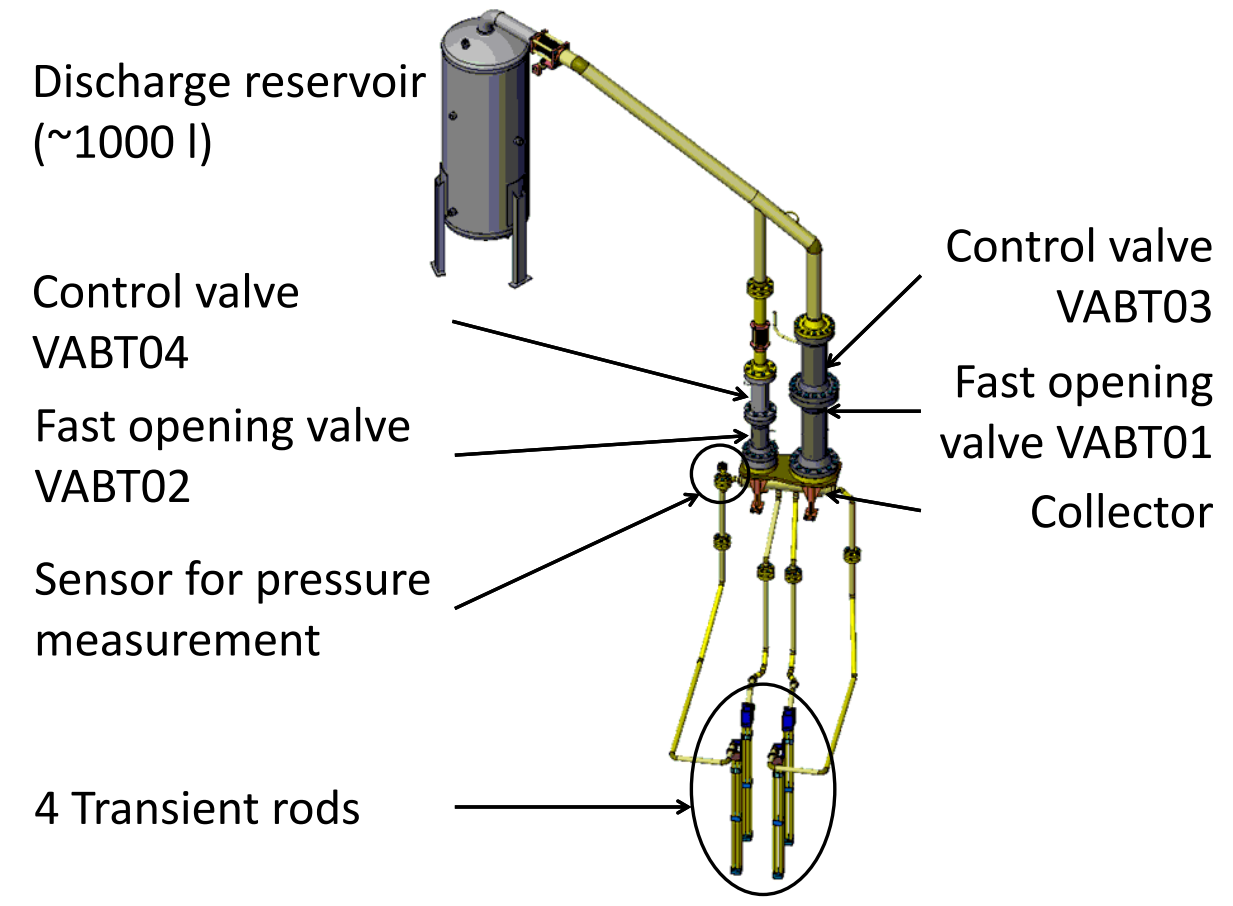

Figure 2: CABRI transient rods system

increase of the transient pulse FWHM is necessary. This can be done by successively opening the fast opening valves of the low and then the high flow-rate channels, it is named "structured transient" (see comparison on figure 3). The parameters of the transients are described in Table 1.

This paper focuses on the optimization of those parameters to reach target characteristics of the power transients. The first part is dedicated to the material and methods used for this study. Then, we will deal with the optimization process analysis. Afterwards, we will analyze the optimized results for 2 power transients. Finally, a last part deals with the adaptation of this method for experimental purposes.

\section{Material and methods}

\subsection{SPARTE}

SPARTE is a point kinetics code optimized for CABRI power transients calculation. It is based on the DULCINEE (Ritter et al. (2010)) multiphysics code. It deals with reactivity injection calculation, point kinetics resolution, 


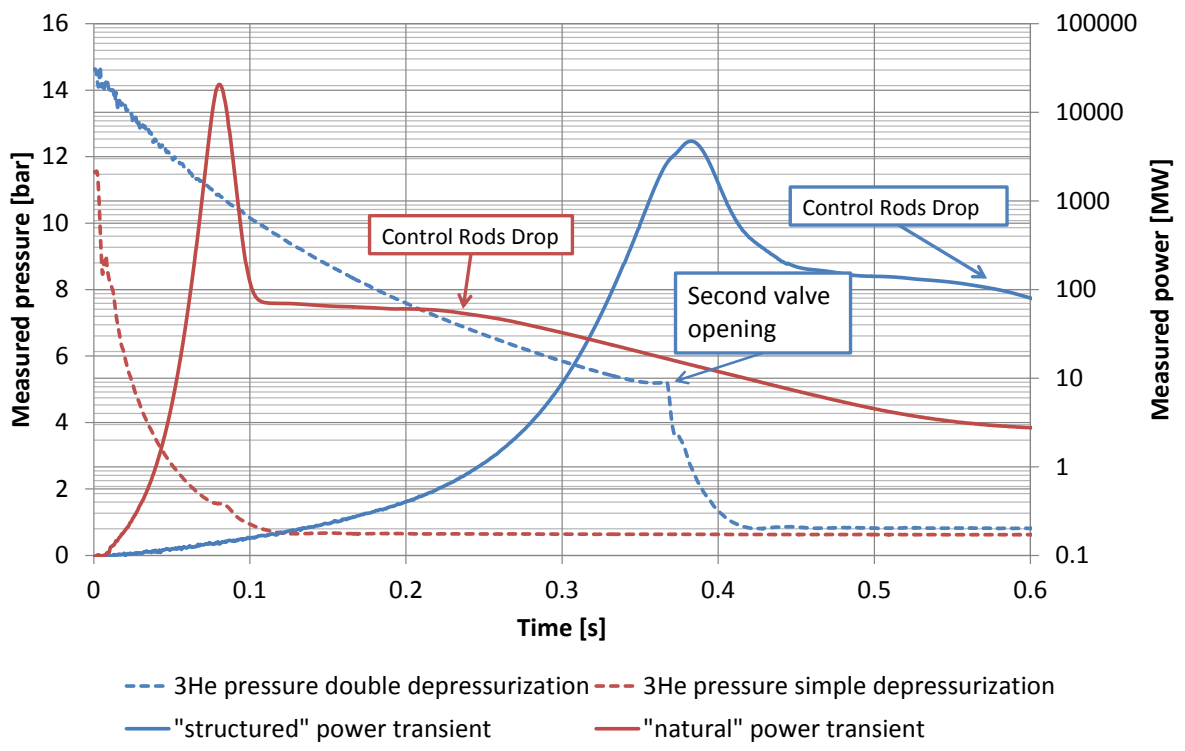

Figure 3: The 2 CABRI power transients types

Table 1: Input parameters of a CABRI power transient

\begin{tabular}{|l|c|r|}
\hline Name & Unit & Description \\
\hline$H 03$ & $m m$ or $\%$ & $\begin{array}{r}\text { Aperture of the control } \\
\text { valve VABT03 }\end{array}$ \\
\hline$H 04$ & $m m$ or $\%$ & $\begin{array}{r}\text { Aperture of the control } \\
\text { valve VABT04 }\end{array}$ \\
\hline$t_{\text {open }}$ & $s$ & $\begin{array}{r}\text { Opening moment of } \\
\text { the fast valve VABT01 }\end{array}$ \\
\hline$P_{0}$ & $b a r$ & Initial ${ }^{3} H e$ pressure \\
\hline$t_{\text {drop }}$ & $s$ & Control rods drop moment \\
\hline
\end{tabular}

temperatures and hydraulics calculation. The geometry is simplified as a single channel with axis-symmetric radial mesh and an axial mesh. Models were added to the code in order to reproduce more realistic CABRI core conditions.

The reactivity injection calculation was improved by studying the ${ }^{3} \mathrm{He}$ 
depressurization by a CFD (Clamens et al. (2018b)) approach and the ${ }^{3} \mathrm{He}$ reactivity function of the density by stochastic neutronics simulations using the TRIPOLI4 code. It has also been validated by an experimental approach during the static neutronics commission tests conducted in 2016. The point kinetic was improved by using a variable effective neutron life time surrogate model deduced from core stochastic neutronics simulations with the TRIPOLI4 (Brun et al. (2011)) French Monte-Carlo code. The reactivity feedbacks calculation was improved by adding a variable Doppler coefficient model (Clamens et al. (2017)) also calculated with the TRIPOLI4 code. For all the neutronics calculations, the JEFF3.1.1 nuclear data library was chosen.

The SPARTE code models also the TOP (Transient Over Power) effect specific to the CABRI power transients. It is a reactivity insertion effect linked to the transient rods ${ }^{3} \mathrm{He}$ heating during the power elevation. The local gas temperature increases of few hundreds degrees implying a faster depressurization in the direction of the valves. All of this leads to a code needing 7 to $70 \mathrm{~s}$ calculation by run (ie one transient simulation) on a Linux $3.4 \mathrm{GHz}$ processor, depending of the complexity of the phenomena involved in the transient.

\subsection{URANIE}

URANIE (Gaudier (2010)) is the CEA's Uncertainties platform. It is a ROOT-based open access code that deals with sampling, code launching, surrogate modeling, uncertainties and optimization. Different optimization methods are available in the URANIE package.

There are 3 types of optimization processes:

- Direct solvers that use regressions to find the optimal parameters,

- Gradient solvers that use local derivatives,

- Genetic Algorithms for multi-criteria optimization.

Genetic algorithms need too many simulations and give many results that we don't need. In this study, gradient and direct solvers are compared. The process is managed by a program generated by an URANIE function. The user defines an objective, constraints, a code to launch, input and output files and the optimization parameters. The optimization starts with a point defined by the user containing optimization parameters. At each step, the 
algorithm launches the SPARTE code with different input files. The algorithm changes the optimization parameters at every step to get closer to the objective with respect of the constraints.

\section{Analysis of the optimization process}

\subsection{Objective determination}

The target characteristics are given in terms of FWHM and energy deposited in the maximum flux plane during the pulse time. This energy is converted to the core energy using a coupling factor and the axial neutron flux distribution of the test rod inside the pressurized water loop. The coupling factor is the ratio between the energy deposit in the core and in the test fuel. It can be calculated using Monte-Carlo simulations or measured using a thermal balance method applied on the core and the test cell.

The coupling factor seems to be sensitive to numerous parameters. An objective is to inject the maximal energy during the pulse and complete the energy deposition by delaying the control rods drop. The rest of the energy is deposited before the rods drop by a core power stabilized near $100 \mathrm{MW}$ (see figure 4). The objective of energy deposit during the pulse can be translated in term of maximal power by assimilating the pulse to a Gaussian function. The FWHM of the Gaussian function is related to the standard deviation $(\sigma)$ by the following relation:

$$
F W H M=2 \cdot \sqrt{2 \ln (2)} \cdot \sigma
$$

The deposited energy is the integral of the Gaussian function and is related to the maximum power by:

$$
E_{\text {core }}=P_{\max } \cdot \sigma \cdot \sqrt{2 \pi}
$$

From (1) and (2), we can deduce a relation linking the maximal power to the FWHM and the energy deposited during the pulse:

$$
P_{\text {max }}=\frac{2 \sqrt{2 \ln (2)} \cdot E_{\text {core }}}{\sqrt{2 \pi} \cdot F W H M} \simeq 0.9394 \cdot \frac{E_{\text {core }}}{F W H M}
$$

Moreover, we observe a slight difference between the power shape and the Gaussian function. The energy deposit evaluated by the Gaussian function 


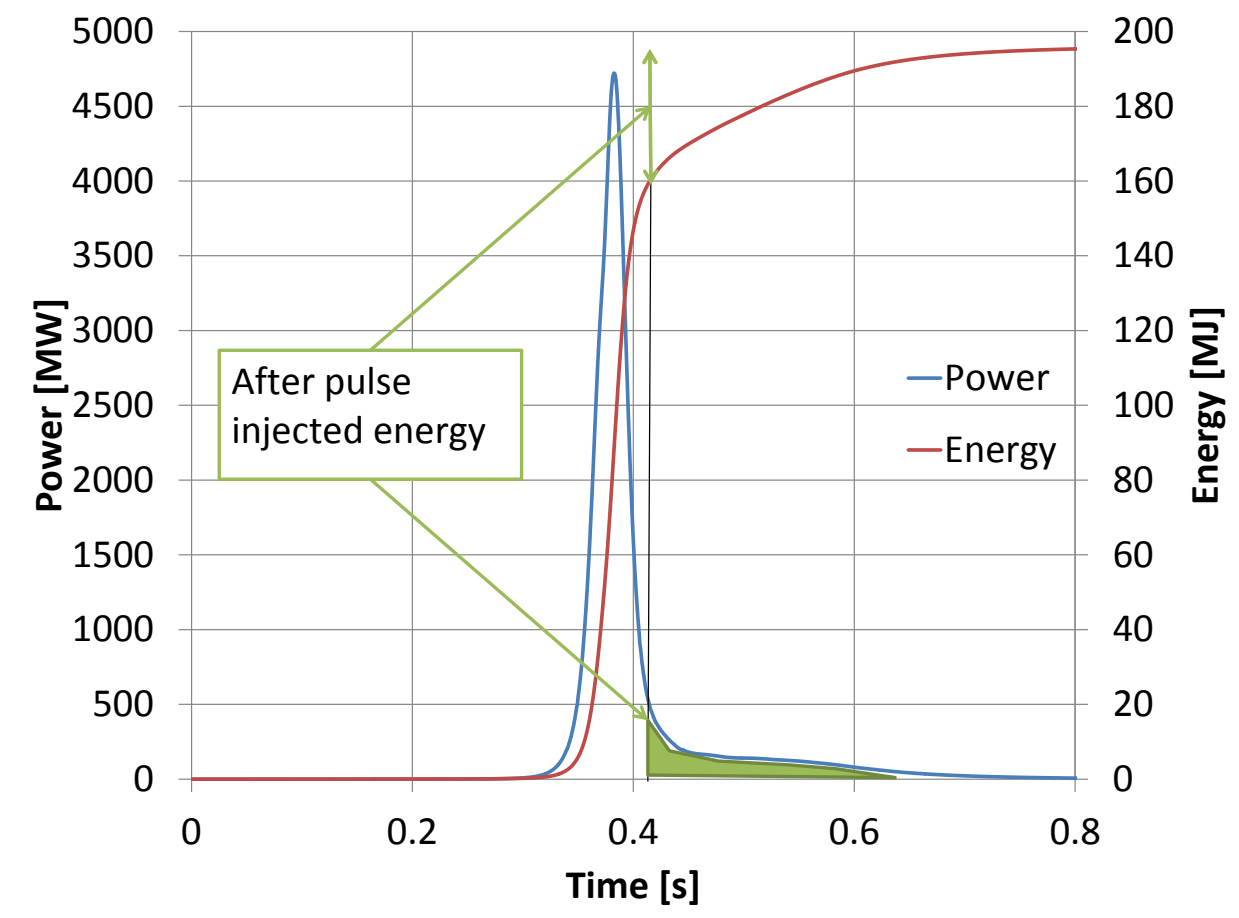

Figure 4: Time dependence of power and energy deposition during a measured CABRI "structured" power transient.

is slightly lower than the real deposit by a factor of $\sim 95 \%$. It means that (3) needs to be multiplied by 0.95 for the optimization process.

$$
P_{\text {ref }} \simeq 0.8924 \cdot \frac{E_{r e f}}{F W H M}
$$

In order to reduce the problem to a mono-objective target, a simple combination function representing the distance to the target characteristics is developed:

$$
\begin{gathered}
y=\frac{\left(F W H M-F W H M_{r e f}\right)^{2}}{F W H M_{r e f}^{2}}+\frac{\left(E_{c o r e}-E_{r e f}\right)^{2}}{E_{r e f}^{2}} \\
+\frac{\left(P_{\max }^{2}-P_{r e f}\right)^{2}}{P_{r e f}^{2}}
\end{gathered}
$$

Where:

- $F W H M_{r e f}$ is the objective FWHM,

- $E_{r e f}$ is the objective total deposited energy (pulse + after), 
- $P_{r e f}$ is the objective maximum power defined by (4).

The 3 terms of (5) are not used in all cases, it depends on the test objectives.

This type of problem has an infinite number of solutions. In order to ensure the best convergence, the problem must be limited to 3 input parameters to be optimized. If the convergence is not sufficient at the end of the first optimization, the starting point is replaced by the last point calculated and the process is relaunched. Then, the input step size is larger, and the algorithm converges to a more accurate solution. 4 algorithms will be rapidly presented and later compared on the test cases. They are implemented in the NLopt library. We are searching a compromise between execution speed and precision of the response.

\subsection{The COBYLA method}

Constrained optimization by linear approximation (COBYLA) is a direct optimization method developed by Michael Powell (Powell (1989)). The method searches a vector (input parameters) so that the applied function (here SPARTE) is minimal or maximal. It is a step-by-step algorithm that learns from past steps to make a linear regression. At every step, the input step size is reduced until it reaches a minimal value defined by the users and marks the end of the optimization process.

\subsection{The BOBYQA method}

The Bobyqa algorithm has also been developed by Michael Powell (Powell (1989)). It is very similar to the Cobyla methodology as a direct solver. The Bobyqa algorithm uses a quadratic models for interpolation. This algortihm is faster than Cobyla but does not always give physical results.

\subsection{The Nelder-Mead method}

The Nelder-Mead method is a non linear algorithm (Nelder and Mead (1965)). It is also called downhill simplex method, it uses the simplex concept in a $\mathrm{N}$ dimensions space. The simplex is transformed during iterations in order to minimize a function.

\subsection{The Subplex method}

The Subplex method is a variant of Nelder-Mead as it uses it on a sequence of subspaces developed by T. Rowan( Rowan (1990)). This method is claimed to be much more efficient and robust than the original Nelder-Mead. 


\subsection{Uncertainties propagation}

Two types of uncertainties are differentiated in the problem:

- Parameters uncertainties linked to the instrumentation precision (see table 2). The propagation of those uncertainties through the SPARTE code gives the reproducibility of the transient. All parameters are considered uncorrelated.

Table 2: Uncertain input parameters for power transients calculations (1 RMS)

\begin{tabular}{|l|r|}
\hline Name & $\sigma$ \\
\hline Aperture - $H 03$ & $0.12 \mathrm{~mm}$ \\
\hline Aperture - H04 & $0.06 \mathrm{~mm}$ \\
\hline Opening moment of the VABT01- $t_{\text {open }}$ & $1 \mathrm{~ms}$ \\
\hline Initial Pressure of the ${ }^{3} \mathrm{He}-P_{0}$ & $1 \%$ \\
\hline Purity of the ${ }^{3} \mathrm{He}($ mean $=99 \%)-$ pur & $0.25 \%$ \\
\hline Initial system temperature - $T_{0}$ & $0.5 \mathrm{~K}$ \\
\hline Initial power - power & $1 \%$ \\
\hline Rod drop instant $-t_{\text {drop }}$ & $2 \mathrm{~ms}$ \\
\hline
\end{tabular}

- Models uncertainties linked to the precision of the calculations and modelling hypothesis (see table 3). The propagation of those uncertainties through the SPARTE code gives the prediction uncertainty.

All the design of experiments are made following a normal distribution of the uncertain parameters. The analysis of the uncertainties are made by using the Sobol indexes method. It consists in calculating the variability of one output depending on the different inputs variabilities. There are first order indexes (6) that evaluate the variance of the output quantity depending on the variability of one input alone.

$$
S_{i}=\frac{\operatorname{Var}_{X_{i}}\left(E_{X_{\sim i}}\left(Y \mid X_{i}\right)\right)}{\operatorname{Var}(Y)}
$$

However, some variables are linked to each others and have impact on the output if their variations are simultaneous. Those variations are calculated 
Table 3: Uncertain models for power transients calculations (1 RMS)

\begin{tabular}{|l|r|}
\hline Name & $\sigma$ \\
\hline $\begin{array}{l}\text { Effective Delayed Neutrons fraction } \\
\beta_{\text {eff }} \text { (beta) }\end{array}$ & $23 \mathrm{pcm}$ \\
\hline $\begin{array}{l}\text { Prompt Neutron generation time } \\
\Lambda_{\text {eff }} \text { (dlamb) }\end{array}$ & $1.25 \mu \mathrm{s}$ \\
\hline $\begin{array}{l}{ }^{3} \text { He reactivity } \\
\Delta \rho \text { (deltrho) }\end{array}$ & $4 \%$ \\
\hline $\begin{array}{l}\text { Density at the end of the } \\
\text { depressurization - (dfinal) }\end{array}$ & $5 \%$ \\
\hline $\begin{array}{l}\text { Integral Doppler coefficient } \\
(d d o p)\end{array}$ & $10 \%$ \\
\hline $\begin{array}{l}\text { Deviation of the depressurization } \\
\text { rate by TOP effect - (dtop) }\end{array}$ & $5 \%$ \\
\hline $\begin{array}{l}\text { Depressurization characteristic } \\
\text { time - (dtdepr) }\end{array}$ & $5 \%$ \\
\hline
\end{tabular}

by the interaction Sobol indexes with $\mathrm{n}$ degrees representing $\mathrm{n}$ variables. The total order index (7) measures the contribution to the output variance of $X_{i}$, including all variances caused by its interactions, of any order, with any other input variables.

$$
S_{T i}=\frac{E_{X_{\sim i}}\left(\operatorname{Var}_{X_{i}}\left(Y \mid X_{\sim i}\right)\right)}{\operatorname{Var}(Y)}
$$

Where an estimator is:

$$
\begin{gathered}
E_{X_{\sim i}}\left(\operatorname{Var}_{X_{i}}\left(Y \mid X_{\sim i}\right)\right) \simeq \\
\frac{1}{2 N} \cdot \sum_{j=1}^{N}\left(\left(f\left(A_{B}^{i}\right)_{j}-f(A)_{j}\right)\right)^{2}
\end{gathered}
$$

In URANIE, the Sobol indexes are calculated by the Saltelli (Saltelli (2002)) method that reduces the number of code assessment for the same precision compared to the original Sobol method. More informations about the uncertainties propagation on CABRI power transient are available in (Clamens et al. (2018a)). 


\section{The optimization results}

Two types of power transients are part of the CIP program: "natural" and "structured" transients. First ones deposit a lot of energy with an approximate $10 \mathrm{~ms}$ FWHM. Those transients aim at studying the cladding failure and the fission products release inside the pressurized water loop. The "structured" transients reproduce the real conditions of a control rod ejection in a PWR leading to a 30 ms (Jernkvist and Massih (2010)) FWHM power transient.

\subsection{First case: "natural" transient}

This paragraph will focus on the optimization of an hypothetic power transient with a 220 MJ total energy deposit with $50 \%$ of this energy deposited during the pulse. The input parameters to optimize are the aperture of the high flow rate channel control valve (H03), the initial ${ }^{3} \mathrm{He}$ pressure (P0) and the control rods drop moment (tdrop). The combination function described in (5) is simplified by the FWHM term that is not an objective for this test. We resume in table 4 the efficiency of the 4 different optimization algorithms to resolve this case.

Table 4: Comparison of the different optimization algorithms

\begin{tabular}{|c|c|c|}
\hline Method & Processing time & combination \\
\hline COBYLA & $19 \mathrm{~min}$ & 0.0014 \\
\hline BOBYQA & $6 \mathrm{~min}$ & 0.0282 \\
\hline Nelder-Mead & $31 \mathrm{~min}$ & 0.0003 \\
\hline Subplex & $47 \mathrm{~min}$ & $5.9 \cdot 10^{-8}$ \\
\hline
\end{tabular}

Table 4 shows that the Subplex algorithm gave the best minimization of the combination function. It is also the longest method to reach a result, but it gives a very well converged solution in only one processing. The different methods tend to converge to the same optimized parameters with different precisions on the characteristics (see figure 5), while they all started with the same point (H03, P0, tdrop).

The measurement and calculation uncertainties have been propagated through the SPARTE code with central value parameters coming from the Subplex algorithm. 800 calculations serve as a base for reproducibility evaluation and 900 for calculation uncertainties evaluation. The input parameters 


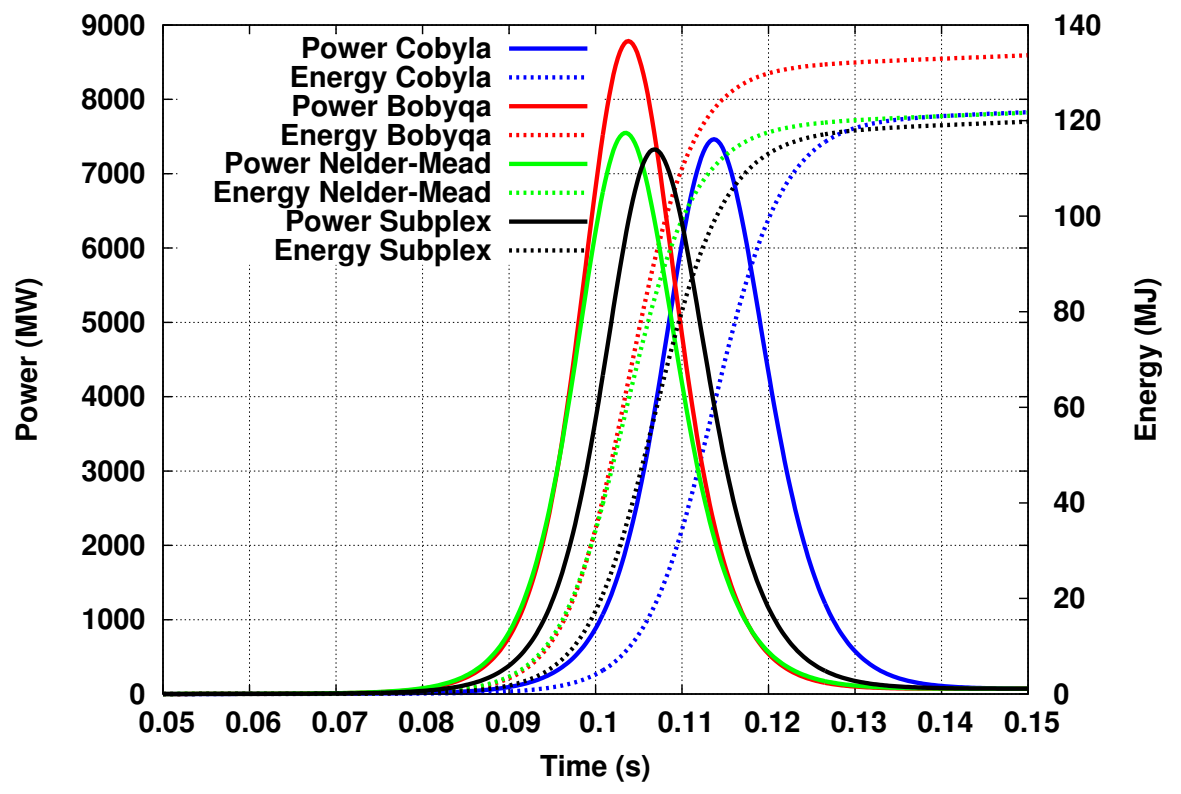

Figure 5: Comparison of the "natural" power transients shapes

are sampled by a Sobol sequence DOE in order to compute as well the Sobol indexes associated to the inputs. The results of those uncertainties propagations are resumed in table 5 .

Table 5: Results of uncertainties propagation for the "natural transient"

\begin{tabular}{|c|c|c|c|}
\hline & $P_{\max }$ & $E_{\text {core }}$ & FWHM \\
\hline$\sigma_{\text {exp }}$ & $1.8 \%$ & $0.7 \%$ & $1.0 \%$ \\
\hline$\sigma_{\text {calc }}$ & $12.9 \%$ & $8.9 \%$ & $5.9 \%$ \\
\hline
\end{tabular}

The reproducibility is very good for this type of transient, it was experimentally proved during the commission tests in the first trimester of 2017 . The uncertainty associated to the models represents the uncertainties on the prediction of the transient. It is higher than the experimental uncertainty and should be analyzed by calculating the Sobol sensitivity indexes.

Figure 6 shows the sensitivity Sobol indexes associated to the model uncertainties for the maximum power. The uncertainty on the maximum power 


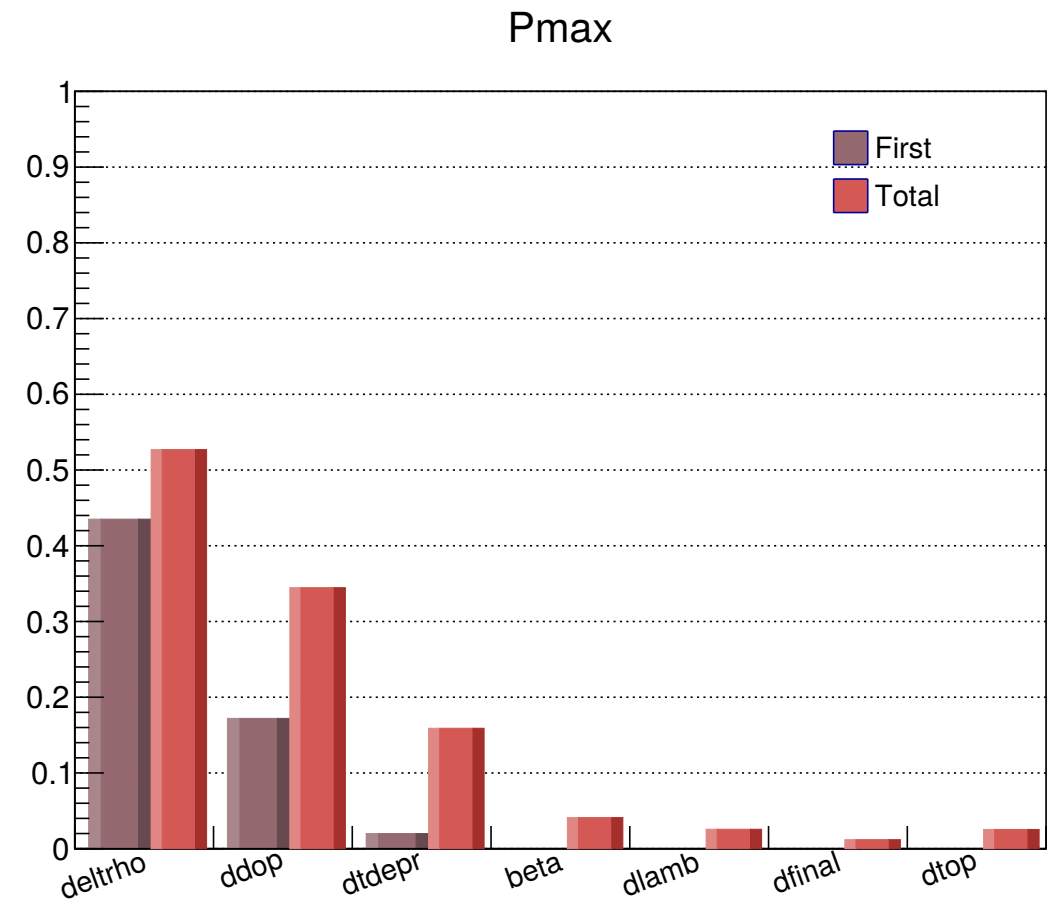

Figure 6: Sobol sensitivity indexes for $P_{\max }$ of the "natural" transient

is mainly due to the uncertainties on the ${ }^{3} \mathrm{He}$ reactivity (deltrho), on the Doppler coefficient (ddop) and on the depressurization speed (dtdepr).

Globally the total order parameters are really high. The depressurization speed is strongly related to the other models in the calculation of the injected reactivity, particularly the ${ }^{3}$ reactivity model. The Doppler coefficient is related to the fuel temperature in the calculation of the Doppler reactivity feedback. It depends on the core power and consequently of the other parameters.

\subsection{Second case: "structured" transient}

A "structured" transient is made by successively opening the low flowrate channel and the high flow-rate channel. It allows the deposition of a lot of energy in a larger and less powerful (ie "peaked") transient. Two input parameters are added to the optimization process: the aperture H04 and the fast valve opening time. The aperture of the large control valve H03 is fixed to its maximum. 
Three apertures of the small control valve are fixed (40, 50 and $60 \%$ of the maximal cross section) in order to compare different solutions. The optimization process consists in searching the good initial ${ }^{3} \mathrm{He}$ pressure, fast valve opening time and control rods drop instant to reach the target $130 \mathrm{MJ}$ of energy deposit during the pulse with a $30 \mathrm{~ms}$ FWHM. The $130 \mathrm{MJ}$ deposit must represent $75 \%$ of the total transient energy. So the combination function is not simplified in this case. The different optimization methods were also compared on the "structured" transient case. The subplex method has also been selected. In order to accelerate the process, the total energy deposit is not integrated to the combination function as the control rods drop instant is no more a parameter to optimize in a first approach. The objective is to focus in a first approach on the power transient shape. The control rods drop instant is optimized in a second run as the only parameter (very fast $\sim 5 \mathrm{~min})$. The results of the optimization are resumed in table 6 .

Table 6: Uncertain models for power transients calculations

\begin{tabular}{|c|c|c|c|c|c|}
\hline H04 & $\begin{array}{c}P_{0} \\
(\mathrm{bar})\end{array}$ & $\begin{array}{c}t_{\text {open }} \\
(\mathrm{ms})\end{array}$ & $\begin{array}{c}t_{\text {drop }} \\
(\mathrm{ms})\end{array}$ & $\begin{array}{c}\text { FWHM } \\
(\mathrm{ms})\end{array}$ & $\begin{array}{c}\mathrm{P}_{\max } \\
(\mathrm{MJ})\end{array}$ \\
\hline $40 \%$ & 10.8 & -8.6 & 331 & 29.4 & 3980 \\
\hline $50 \%$ & 12.0 & -5.7 & 244 & 29.5 & 3910 \\
\hline $60 \%$ & 9.2 & -7.1 & 281 & 30.1 & 3650 \\
\hline
\end{tabular}

The opening instant $\left(t_{\text {open }}\right)$ of the fast valve of the high flow-rate channel is relative to the instant of the peak generated by single opening of the low flow-rate channel. By opening the high flow-rate channel few milliseconds before the peak, the energy deposit is optimized. According to the study, we are able to realize the optimal transient with different apertures of the control valve by adjusting the initial ${ }^{3} \mathrm{He}$ pressure and the opening instant. The total energy deposit can be adjusted by mastering the control rods drop instant.

The analysis of the power curves and energy curves (see figure 7) does not allow us to clearly identify a set of parameters. Every transient modeled presents both a good shape and a good timing for energy deposition. We will identify the best power transient by propagating models and experimental uncertainties. A good compromise between prediction precision and reproducibility must be selected for the test. 


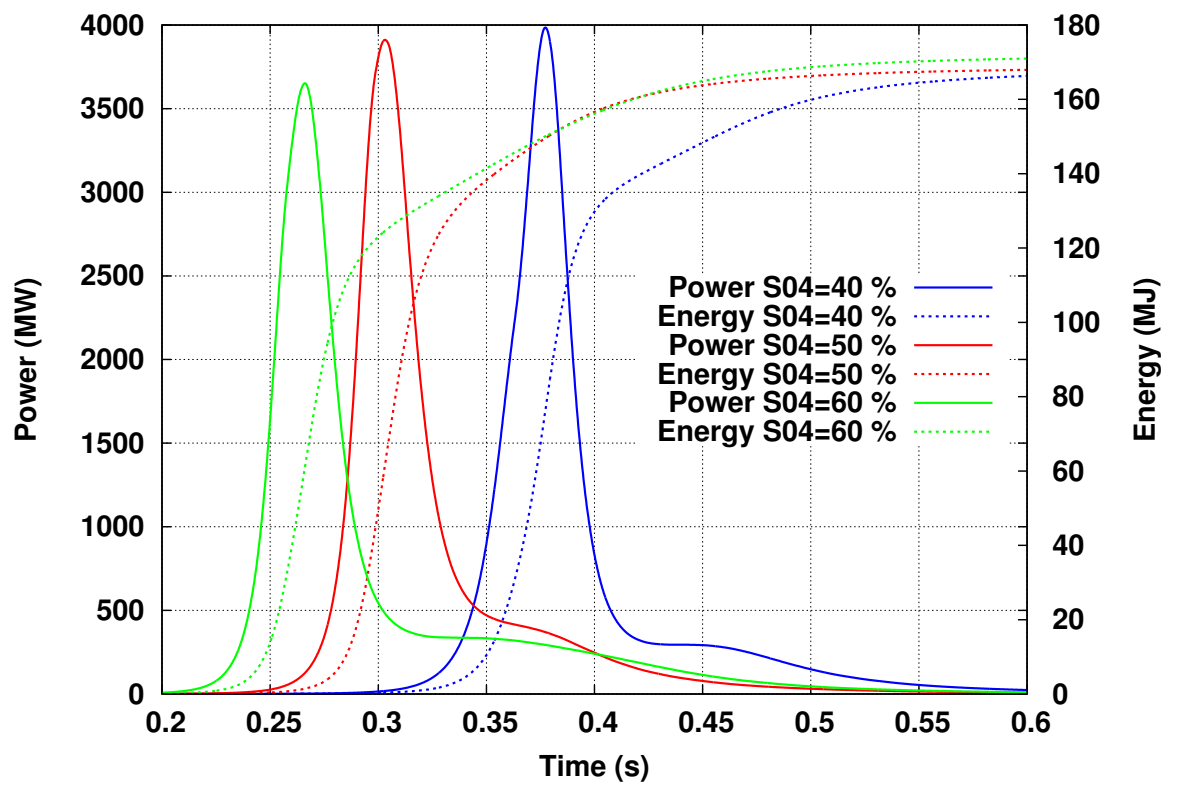

Figure 7: Comparison of the "structured" power transients shapes

Table 7: Uncertainties propagation for "structured" transients

\begin{tabular}{|c|c|c|c|c|}
\hline \multirow{2}{*}{ H04 } & \multicolumn{2}{|c|}{ FWHM } & \multicolumn{2}{c|}{ Energy $(1.2 \mathrm{~s})$} \\
& $\sigma_{\exp }$ & $\sigma_{\text {calc }}$ & $\sigma_{\text {exp }}$ & $\sigma_{\text {calc }}$ \\
\hline $40 \%$ & $11.7 \%$ & $5.0 \%$ & $1.3 \%$ & $11.2 \%$ \\
\hline $50 \%$ & $3.7 \%$ & $12.4 \%$ & $1.2 \%$ & $11.5 \%$ \\
\hline $60 \%$ & $5.1 \%$ & $8.5 \%$ & $0.8 \%$ & $9.5 \%$ \\
\hline
\end{tabular}

The uncertainties on the power transient characteristics linked to calculation and parameters uncertainties are presented in table 7 . They are obtained by the propagation method. The uncertainty on the energy deposit is more linked to the models uncertainties as for the "natural transient". Two phenomena are observable by analyzing the uncertainties on FWHM in the table 7. For 1 transient, we observe a large uncertainty on FWHM from models uncertainties propagation, and a small uncertainty from experimental uncertainties propagation. It means that the $\mathrm{H} 04=50 \%$ presents a good reproducibility, but without a satisfactory confidence in the FWHM 
calculation.

For another transient, the reproducibility is worse but the calculation reliability is better. Moreover, experimental investigations have already been performed with this aperture. The safest choice for the experimentalists is the first case with the $40 \%$ aperture of the low flow-rate channel control valve thanks to its relatively small uncertainty on FWHM caused by the models.

The $60 \%$ case presents the minimal total uncertainty. The $60 \%$ aperture solution seems attractive since the reproducibility is really better than the $40 \%$ aperture solution. This solution should be tested during start-up phases without the test device.

As we can see on Figure 8, the uncertainty on the FWHM is mostly due to the opening instant of the second fast valve. The FWHM presents a linear variation in function of the opening instant around the nominal value.
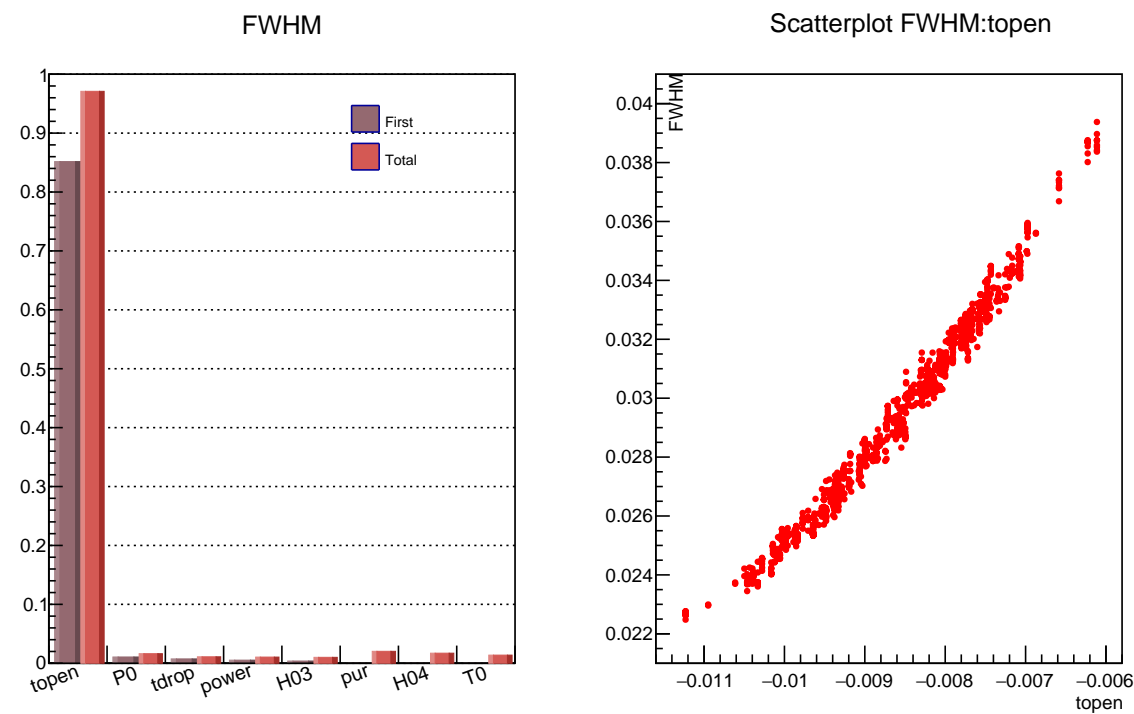

Figure 8: Sobol sensitivity indexes for $F W H M$ of the "structured" transient and fast valve opening instant (topen) dependency of the FWHM

\section{Discussion}

In this section, we deal with the analysis of the results and advises for the experimental approach. 
Four optimization algorithms have been tested for the prediction of the CABRI power transients. Although it is the longest to execute, the Subplex method was selected thanks to its precision compared to the others. The other methods can also converge to a solution but need to be relaunched few times with the last parameters to converge to the same precision. The Subplex method does this process itself by involving the Nelder-Mead algorithm. That is why this method is more practical for the user.

The optimization method works well for both "natural" and "structured" power transients. Valves apertures, initial pressure and opening timing influence the power transient shape $\left(\mathrm{FWHM}, \mathrm{P}_{\max }\right)$ whereas control rods drop time is just adjusting the total energy deposit. Hence, those parameters can be separated for a more accurate solution and a shorter calculation time. Fixing 3 discrete control valve apertures allows us to focus on different solutions and compare them through uncertainties propagation.

The prediction uncertainties are computed by propagation of the models uncertainties. Those uncertainties have to be taken into account for experimental investigations, particularly when the deposited energy is quite high near the safety limit $(\sim 260 \mathrm{MJ})$. The prediction should be used with some margin according to the total uncertainties obtained by propagation of all the uncertainties (measurements + models). In the case of a "structured" transient, the experimentalist should try different opening instants of the fast valve (4 to 5 transients with opening instants varying from -5 to $+5 \mathrm{~ms}$ around the optimized value). The points will then be used to create an order 2 polynomial function between the FWHM and the opening instant of the fast valve in order to optimize the opening instant and reach the FWHM targeted value. As in the numerical optimization method, the total energy deposit should be adjusted a-posteriori by setting up the control rods drop.

In order to predict the uncertainties on the characteristics of the test transient, the experimentalists perform reproducibility tests. It consists in producing few power transients with the same parameters adjustments. We estimated the reproducibility by propagation of the parameters uncertainties through the SPARTE code. For "natural" transients, a very good reproducibility is calculated in accordance with observations. For "structured" transients, a 5 to $6 \mathrm{~ms}$ uncertainty on the FWHM is expected with the optimized parameters mainly due to the variations of the opening instant of the fast valve. This standard deviation is consistent with the observations on past CABRI power transients (Clamens et al. (2017)). 


\section{Conclusion}

For the RIA tests in the CABRI research reactor, the experimentalists have to arrange the power transient characteristics in terms of FWHM and energy deposited in the test rod. That's why, we search to optimize the parameters of the CABRI reactivity injection system: the ${ }^{3} \mathrm{He}$ transient rods. Since 2016, the SPARTE code is developed and aims to reproduce the CABRI power transients. It is based on a point kinetics approach in which we added surrogate models based on best estimates simulations and experimental analysis.

This study shows that we are able to optimize the CABRI power transients parameters thanks to the SPARTE code and the URANIE uncertainty platform. Among 4 optimization algorithms included in the URANIE platform, we selected the one giving the most precise solution. The Subplex method involves few tenths of SPARTE simulations to optimize the power transient characteristics (FWHM, maximum power, deposited energy) by selecting the driving parameters of the transient rods system values. It works well with "natural" and "structured" transients.

For "structured" transients, the problem that we solved has an infinite number of solutions. We targeted experimental objectives by fixing the control valves apertures of the transients rods system. The selection of a set of parameters can be made by comparison of the uncertainties on the transients characteristics.

The calculation reliability is evaluated by propagation of the models uncertainties. Those uncertainties depend on the transient. The impacts of each input is evaluated by determining the Sobol indexes of first and total order. We calculated standard deviations of about $10 \%$, mainly due to uncertainties on the Doppler coefficient, the depressurization and the ${ }^{3} \mathrm{He}$ reactivity models.

The power transient shape reproducibility can be estimated by propagating the uncertainties on the driving parameters. We compute a very good reproducibility for the "natural" transients in accordance with measurements. For the "structured" transients, the FWHM and the maximum power are very sensitive to the fast valve opening time. It increases the uncertainty on the power transient shape. Measurements confirm a variation of $5 \mathrm{~ms}$ $(\sigma=2.5 \mathrm{~ms})$ of the FWHM around the central value.

In order to maximize the chance to reach the objective FWHM, the experimentalists should test different opening times around the optimized value. 
The results must be used to establish a function linking the fast valve opening time to the FWHM.

\section{Bibliography}

Brun, E., Dumonteil, E., Hugot, F., Huot, N., Jouanne, C., Lee, Y., Malvagi, F., Mazzolo, A., Petit, O., Trama, J., others, 2011. Overview of TRIPOLI4 version 7, Continuous-energy Monte Carlo Transport Code.

Clamens, O., Blaise, P., Hudelot, J.-P., Lecerf, J., Biard, B., Apr. 2018a. Uncertainty assessment on the prediction of the CABRI power transients. In: Proc. PHYSOR2018. Cancun, Mexico.

Clamens, O., Couybes, J., Lecerf, J., Hudelot, J.-P., Duc, B., Pantera, L., Blaise, P., Biard, B., Jun. 2017. Analysis of the CABRI power transients - Prediction improvements using a combination of measurements and calculation. In: Proc. Int. Conf. ANIMMA2017. Liege.

Clamens, O., Lecerf, J., Hudelot, J.-P., Duc, B., Cadiou, T., Blaise, P., Biard, B., 2018b. Assessment of the 3He pressure inside the CABRI transient rods - Development of a surrogate model based on measurements and complementary CFD calculations. EPJ Web of Conferences 170, 04005 .

URL https:/articles/epjconf/abs/2018/05/epjconf_animma2018_04005/epjconf_animma2

Gaudier, F., Jan. 2010. URANIE: The CEA/DEN Uncertainty and Sensitivity platform. Procedia - Social and Behavioral Sciences 2 (6), 7660-7661. URL http://www.sciencedirect.com/science/article/pii/S1877042810013078

Jernkvist, L. O., Massih, A. R., 2010. Nuclear Fuel Behaviour under Reactivity-initiated Accident (RIA) Condition: State-of-the-art Report. Nuclear Energy Agency, Organisation for Economic Co-operation and Development (OECD). URL https://dspace.mah.se/handle/2043/10054

Nelder, J. A., Mead, R., Jan. 1965. A Simplex Method for Function Minimization. The Computer Journal 7 (4), 308-313.

URL https://academic.oup.com/comjnl/article/7/4/308/354237 
Powell, M. J. D., Aug. 1989. A tolerant algorithm for linearly constrained optimization calculations. Mathematical Programming 45 (1-3), 547-566. URL https://link. springer.com/article/10.1007/BF01589118

Ritter, G., Gueton, O., Mellier, F., Beretz, D., Oct. 2010. Neutron Commissioning in the New CABRI Water Loop Facility. IEEE Transactions on Nuclear Science 57 (5), 2697-2701.

Rowan, T. H., 1990. Functional stability analysis of numerical algorithms.

Saltelli, A., May 2002. Making best use of model evaluations to compute sensitivity indices. Computer Physics Communications 145 (2), 280-297. URL http://www.sciencedirect.com/science/article/pii/S0010465502002801 